\section{A CASE STUDY ON RESILIENCE AGAINST PSYCHOLOGICAL IMPACT - FOCUSING ON THE MEANINGS OF 'WORK'-}

${ }^{1} \mathrm{~K}$ Otani ${ }^{*},{ }^{2} \mathrm{Y}$ Matsuda, ${ }^{3} \mathrm{E}$ Tomizawa, ${ }^{4} \mathrm{M}$ Negishi, ${ }^{5} \mathrm{M}$ Arakida. ${ }^{1}$ Japanese Red Cross Toyota College of Nursing, Toyota Aichi, Japan; ${ }^{2}$ International University of Health and Welfare Graduate School, Minato-ku Tokyo, Japan; ${ }^{3}$ Sikoku University, Tokushima Tokushima, Japan; ${ }^{4}$ Fujisawa Taxi Co., LTD., Fujisawa Kanagawa, Japan; ${ }^{5}$ International University of Health and Welfare, Odawara Kanagawa, Japan

\subsection{6/oemed-2018-ICOHabstracts.1518}

Introduction Resilience is a personal characteristic that assists recovery from psychological impact. This study aimed to examine resilience-related factors focusing on the meanings of 'work'.

Methods This was a case study. A semi-guided interview was conducted with a participant in December 2014. We gathered narratives and analysed qualitatively and descriptively. Our University ethics committee approved this study.

Results The participant was a woman in her 30 s. The source of psychological impact was a surgery to remove uterine cancer in her $20 \mathrm{~s}$. Her current work involved giving lecturers on the importance of medical examination. The interview lasted 64 min. From the interview, 102 codes, 42 sub categories, and 11 categories were obtained. The extracted items included decline of self-esteem, acceptance of one's situation, existence of supporters, sorting out feelings through work, clarification of one's role, and being an advocator of uterus cancer.

Discussion Resilience after experiencing psychological impact was related to work and clarification of one's role through the work. Working plays a significant role in supporting one's resilience. It is necessary to facilitate environmental system that achieves good balance between work and treatment.

\section{HOW JUNIORS DOCTORS LIVE THIER WORK DURING PREGANCY? A QUALITATIVE STUDY IN FRANCE}

Ménage Alexandra, Loddé Brice, Chiron Benoît, Dewitte Jean-Dominique, Pougnet Richard. Teaching Hospital Of Brest, Brest, France

\subsection{6/oemed-2018-ICOHabstracts. 1519}

Introduction During the end of their training, the injections are subjected to much stress. The prevalence of Burnout syndrome is near $30 \%$ in the literature. Some junior doctors get pregnant during this period. And pregnancy involve physical and psychological changes in women. How do these women live their work as doctors? The aim of this article is to assess the feelings of these womens.

Matrial and method it was a qualitative study. It was based on semi-directed interviews. The analysis was carried out according to the model of the Grounded theory.

Results 10 interviews were carried out, saturation of the data was obtained from the 8 th interview. The variation was maximal at the 10th interview. The analysis of the data revealed four main themes: parenthood, work, industrial relations and adaptation.

Conclusion Becoming a mother during the end of medical studies represents an additional difficulty for the women. This requires adaptability to limit the negative consequences, both professionally and personally.

\section{2 OPPORTUNITIES FOR WORKSITE-BASED CERVICAL CANCER SCREENING AMONG FEMALE FIREFIGHTERS}

1,2 Natasha Schaefer Solle* ${ }^{2,3}$ Alberto J Caban-Martinez, ${ }^{2,3}$ David J Lee, ${ }^{1,2}$ Erin N Kobetz. ${ }^{1}$ Department of Medicine, University of Miami, Miller School of Medicine, Miami, FL, USA; ${ }^{2}$ Sylvester Comprehensive Cancer Centre, University of Miami, Miami, FL, USA; ${ }^{3}$ Department of Public Health Sciences, University of Miami, Miller School of Medicine, Miami, FL, USA

10.1136/oemed-2018-ICOHabstracts. 1520

Introduction Recent studies have documented differences in cancer rates between female and male U.S. firefighters, including cervical cancer. Despite heightened concern that firefighters may have an increased cancer risk, few studies have examined the cancer prevention strategies pertinent to female firefighters. Cervical cancer is among one of the most common cancers diagnosed in this cohort. Screening underutilization likely accounts for most of the observed, excess cervical cancer risk. This study aims to circumvent the barriers of shift work with the implementation of a Human Papillomavirus (HPV) self sampler in a sample of active female firefighters in Florida, United States.

Methods Female firefighters were recruited from their departments during station visits and cancer eductaion seminars. Women completed eligibility screeners and informed consent prior to participation. If eligible, women received a HPV selfsampler to complete at home or at the fire station. Specimens were returned to the lab for analysis. HPV results were given over the telephone and those who screened HPV positive were referred for follow-up care with their primary physician. Result A total of 251 female firefighters were screened for eligibility, of whom $31(12.3 \%)$ were eligible and interested in participating. Self-sampling kits were sent to 31 of the eligible subjects; $14(45.0 \%)$ participants returned the kits, and 1 (7.0\%) received a positive HPV result. Particpants felt selfsampling was easy to self-administer and would repeat the use of the self-sampler as per national screening guidelines.

Discussion Female firefighters who completed the self sampling kit in this pilot study reported preference for work-site based education and screening options as compared to a traditional women's health exam with a healthcare provider. Further strategies to increase cancer screeining among female firefighters engaging in shift work may be helpful in reducing the cervical cancer burden in the fire service.

\section{THE IMPACT OF HIGH NOISE EXPOSURE ON TEXTILE WORKERS HEALTH - A RETROSPECTIVE 3 YEARS STUDY IN A TEXTILE FACTORY}

${ }^{1}$ RM Stamatin*, ${ }^{2} \mathrm{C}$ Petrea. ' 'A. Popescu' Emergency Military Hospital, Focşani, Romania; ${ }^{2}$ National Sanitary Veterinary Authority - Vrancea County, Focsani, Romania

10.1136/oemed-2018-ICOHabstracts.1521

Introduction Noise pollution is an often source of environmental stress that can increase the risk of important health conditions, including: hearing loss, stress, sleep disturbances or altering sleep wake balance, heart disease. A 5 decibel noise reduction could reduce the prevalence of high blood pressure and coronary heart disease, as well as the annual major economic savings.

Methods We monitored 640 textile workers (617 women, caucazians), on the occasion of the annual medical examination, 
over a three-year period. Audiograms were made to all employees, at the same time with official determination of the noise level, carried out by the Public Health Authority of the county. In selected cases, the workers were also led to specialised examinations (cardiology, neurological, psychiatric, ENT).

Results Official noise level determinations have shown overtaking in many of the technological process points (ironing presses, buttonhole machines, edge banding, staple machines etc.). In situations where workers were rotated at short intervals (30 days), they showed minimal symptoms of high-loud exposure. In situations where workers had fixed workstations, after three years of exposure, a decrease with $5-10 \mathrm{~dB}(\mathrm{~A})$ in auditory acuity was observed, associated with sleep disturbances, nervousness and irritability, a slight increase in blood pressure, at about $20 \%$ of the employees (especially if they were over 50 years old and worked in the noise before). During week-ends and holidays, everyone was feeling good.

Discussion The textile industry is an important sector of concentration of professional high-noise exposure pathology. Technical and organisational measures (risk recognition, wearing individual hearing protection equipment, resting in quiet rooms, separation of noise-generating technology processes, high-tech equipment), as well as medical ones (decrease in headphones use, health education, thorough medical checks), lead to better management of the negative impact of human health noise.

\section{FEELING STRAINED BY THE EXPECTATION TO BE AVAILABLE - GENDER DIFFERENCES OF EMPLOYEES IN SMALL AND MEDIUM-SIZED ENTERPRISES}

A Voss* ${ }^{*}$ W Fischmann, N Amler, E Wischlitzki, J Kiesel, H Drexler. Friedrich-AlexanderUniversität Erlangen-Nürnberg, Germany

\subsection{6/oemed-2018-ICOHabstracts. 1522}

Introduction The increasing use of digital media such as mobile phone, e-mail, messenger services etc. enable a permanent availability of employees, which may lead to mental strain. This study focuses on possible gender-specific differences in feeling strained by the expectation to be permanently available for colleagues, superiors, or customers.

Methods A psychological risk assessment has been carried out with 933 employees within the framework of a collaborative project ('RegioKMUnet'), funded by the German Federal Ministry of Education and Research. Data were collected from October 2016 to June 2017 in 10 small and medium-sized enterprises by paper and online surveys. The basic question was if the respondents had to be short-termed available during their free time or working time and whether that led to mental strain. Data were analysed using IBM SPSS 24.

Results The sample consisted of 569 male and 364 female respondents. $85.6 \%$ of the males and $80.1 \%$ of the females reported they had to be available during their working time. The expected availability led to mental strain for $59.2 \%$ of the men and $50.3 \%$ of the women. During free time $32 \%$ males and $17.5 \%$ females stated they had to be available. For $51.8 \%$ of the men and $37.5 \%$ of the women this led to a feeling of being strained.

A chi-square test confirmed the gender difference of strain during free time $(p=0.042)$.
Conclusion In general the mental strain caused by the expectation of availability is stronger for men, with a significantly higher share during working time. In the free time the distance is even greater but inconclusive due to low case numbers. Additional research is needed to clarify if mental strain caused by availability is confirmedly greater for men. Furthermore possible influence by part-time and full-time working as well as the kind of work activity should be considered.

\section{BREAST FEEDING PRACTICES AMONG WORKING MOTHERS ATTENDING 'POSTNATAL' CLINICS IN TWO HOSPITAL IN KAMPALA, UGANDA}

Khayongo Barbra Clara*. Ministry of Gender, Labour and Social Development, Kampala, Uganda International Commission on Occupational Healt, Allied Health Workers' Association, Kampala, Uganda, Uganda Community and Occupational Health Association

\subsection{6/oemed-2018-ICOHabstracts. 1523}

Introduction Breast milk is critical for proper development of an infant during the first two years of life. The increased rate of women's participation in the labour force has resulted in a 'double work day' which results in a burden of having to accomplish both reproductive and productive roles. The Employment Act 2006, maternity leave is 12 weeks. This is insufficient as it's below recommended 6 months of exclusive breastfeeding thus maternity protection is left to the discretion of the employer and breastfeeding working mothers.

Method A mixed study design using both quantitative and qualitative data collection and analysis methods was used.

Results 232 questionnaires were administered to working mothers attending 'postnatal clinics. 23 KI guides were administered to key decision managers purposively selected from 23 institutions where primary respondents worked. Whereas 224 (96.5\%) were granted maternity leave a proportion of 8 $(3.5 \%)$ were not granted maternity leave. During day time, on demand breastfeeding (over 10 times) was practiced by 225 $(97 \%)$ respondents before resuming full time work. This reduced drastically on resuming full time work to half (between 5 to 7 times during the day) for those in selfemployed and service sectors and only twice for those in Government Ministries, Departments, Agencies, NGO, Schools and banks. $53(22.8 \%)$ breastfed their babies at workplace of these $46(86.8 \%)$ said that the babies were simply laid within working station not designated as a workplace day care centre.

Discussion Whereas we continue to advocate for exclusive breastfeeding, we need to consider the effect of productive roles experienced by working mothers who resume work before their babies are 6 months thus interrupt breastfeeding pattern. Work places in informal and formal sectors had no provisions for nursing mothers to care for their babies nor express their breast milk.

\section{HOUSEWIVES AND MEXICAN WOMEN AT WORK: HEALTH AND PSYCHOLOGICAL FACTORS}

L Fernando Arias-Galicia*. Centro de Investigaciones Interdisciplinarias en Psicología, Universidad Autónoma del Estado de Morelos

10.1136/oemed-2018-ICOHabstracts. 1524 